\title{
Superradiant and stimulated-superradiant emission in prebunched electron-beam radiators. I. Formulation
}

\author{
Avi Gover \\ Faculty of Engineering, Department of Physical Electronics, Tel-Aviv University, Tel-Aviv 69978 Israel
}

(Received 26 February 2004; published 2 March 2005)

\begin{abstract}
A formulation for the characterization of superradiant and stimulated-superradiant radiative emission from bunched electron beams is presented. The radiation is characterized in terms of power and spectral power per radiation mode, which provide a measure of the useful spatially coherent radiation power and spectral power emitted by a radiation source. When the bunched electron beam emits superradiantly, these parameters scale like the square of the number of electrons, orders of magnitude more than spontaneous emission. The formulation applies to emission from single bunches, a finite number of bunches in a macropulse, or periodic bunching. It can be employed on any kind of $e$-beam radiation scheme. Specific analytic expressions are derived for coherent synchrotron radiation and prebunched free-electron laser, providing a basis for comparing and understanding their connection.
\end{abstract}

PACS numbers: 41.60.Cr, 42.50.Fx, 41.60.-m, 52.59.-f

\section{INTRODUCTION}

The goal of this paper is to discuss and analyze within the framework of a unified formulation the enhanced (superradiant) radiation emission processes that can be exhibited in a wide variety of electron-beam radiation schemes and devices, when the electron beam is bunched or density modulated. The discussion is aimed specifically at radiation schemes based on synchrotron radiation emission in a bending magnet [coherent synchrotron radiation (CSR) [1-3]] and at the closely related prebunched beam undulator radiation [prebunched beam free electron laser (PB-FEL) [4-17]], but can be extended as well to SmithPurcell [18] and Cerenkov radiation [19], transition radiation $[20,21]$, and other electron-beam radiation schemes and devices. In all of these radiation schemes, coherent (correlated electrons) and incoherent (uncorrelated electrons) radiative emission processes are possible also without electron bunching by the process of stimulated emission (laser gain), spontaneous emission (shot-noise radiation), and self-amplified spontaneous emission (SASE). Electron beam bunching can give rise in all these schemes to additional radiative emission processes of superradiance $(\mathrm{SR})^{1}$ and stimulated superradiance (STSR), which will be analyzed and reviewed in the following chapters. Note that coherent (superradiant) emission from electron bunches or from the sharp leading edge of an electron beam pulse may be amplified in a long wiggler and enhance the SASE radiation [23-25]. These mixed coherent and incoherent radiation effects may be important

\footnotetext{
${ }^{1}$ The term "superradiance" is sometimes used in FEL theory [14] and in general laser physics in the context of self-amplified spontaneous emission and in the context of amplification of radiation emitted by sharp edge electron pulses that do not satisfy, however, Eq. (2) [16,24]. This is not the connotation meant in this paper. The term superradiance is used here in Dicke's sense only [22].
}

in the timely development of SASE-FEL devices, but are out of the scope of this paper. A formulation relating the coherent and incoherent emission processes in an FEL was presented recently by Pinhasi and Lurie [26].

When an ensemble of discrete emitters of radiation emit independently radiation wave packets, the optical properties of the resultant emitted radiation, such as power, intensity, brightness, and their spectral counterparts, depend on the distribution of the emitters in phase space.

The six-dimensional phase-space volume occupied by a radiation wave packet emitted by a single emitter at some plane $z=$ const along the propagation direction (for freespace propagation $\mathbf{r}_{\perp}, t, \mathbf{k}_{\perp}, \omega$ ) is entirely determined by the radiation scheme and the initial phase-space coordinates of the emitter (e.g., the space-momentum $\mathbf{r}_{e}, \mathbf{p}_{e}$ coordinates of an oscillating electron). Naturally, if the initial distribution of the emitters in space-momentum phase space is spread out, the phase-space distribution of the resultant radiation field of all wave packets will be larger than the phase-space volume of a single wave packet, and therefore the intensity and brightness of the resultant radiation field are reduced. Consequently, the (spectral) power or energy of the radiation that can be collected through given spatial and angular apertures are reduced. In particular, when spatial coherence is required at a fixed frequency $\omega$, only a volume $\pi r_{r} \theta_{r} \cong \lambda / 2$ out of the radiation two-dimensional space-angle $(r, \theta)$ phasespace volume should be collected, and thus the resultant coherent (spectral) power or energy diminishes then (here $\lambda=2 \pi c / \omega$ is the radiation wavelength.

Depending on the radiation scheme, also the total radiative emission power (or energy) may be reduced when the initial phase-space volume of the radiation emitters is spread out. This may be caused (e.g., in the case of radiation from an electron beam) by the expansion of the beam beyond the electron-wave interaction region. In the particular case that the emission process is stimulated (laser 
amplification or oscillation) as in FEL [27], the radiation wave is usually a single transverse mode, which also occupies in transverse two-dimensional space-angle phase-space a volume $\lambda$. To remain within the phase-space volume of the radiation mode, and enable efficient electron-wave interaction, the two-dimensional spaceangle volume of the electron beam, measured by the $e$-beam emittance parameter $\varepsilon=\pi r_{b} \theta_{b}\left(r_{b}\right.$, beam radius; $\theta_{b}$, beam half angular spread), should satisfy the condition [28]

$$
\varepsilon<\lambda
$$

In addition, the electron beam energy spread (or longitudinal velocity spread) should be small enough, so that the Doppler shift spread that it causes to the emission frequency is smaller than the homogeneous broadening, $\Delta \omega_{h}=2 \pi / t_{\mathrm{sp}}$, due to the finite duration $t_{\mathrm{sp}}$ of the radiation wave packet emitted by a single electron.

When the electron beam phase-space distribution spread parameters are kept tight, according to these criteria, the brightness and spectral brightness of spontaneous emission devices, such as undulator-synchrotron radiators [29] [Fig. 1(a)], and the amplification gain and radiative extraction efficiency of stimulated emission devices, such as FEL
[27], are maximized. This maximum is clearly the result of the low entropy of the radiation emitters ensemble (the electron beam), which enables efficient energy conversion into radiation field energy in a minimal phase-space volume. Can this maximum be surpassed? The answer is positive, when considering radiative emission into a specific frequency $\omega$. It involves further ordering of the radiators (electrons) - now in the time domain - by setting their oscillation phase $\varphi_{j 0}$ (or start oscillation time $t_{j 0}$ ) so that they will emit in phase in a given direction or into a given radiation mode.

The process of constructive interference of radiation wave packets emitted by an ensemble of individual classical radiators oscillating in phase is essentially the "superradiant emission" process studied by Dicke in connection with quantum atomic emitters [22]. The total emitted radiation field is then proportional to the number of emitters. Consequently, the power intensity and the radiative energy emitted by the entire ensemble is proportional to the square of the number of emitters.

In the case of electron-beam radiation devices, the superradiance emission condition, requiring oscillation of all electrons at the same phase, is satisfied when

$$
t_{b} \ll 2 \pi / \omega
$$

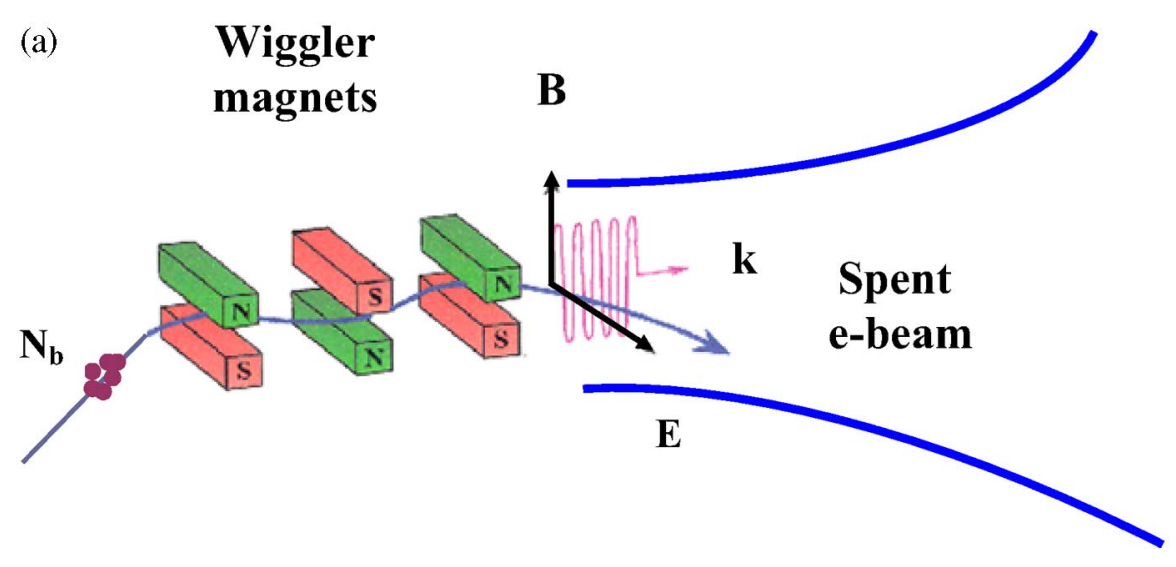

(b)

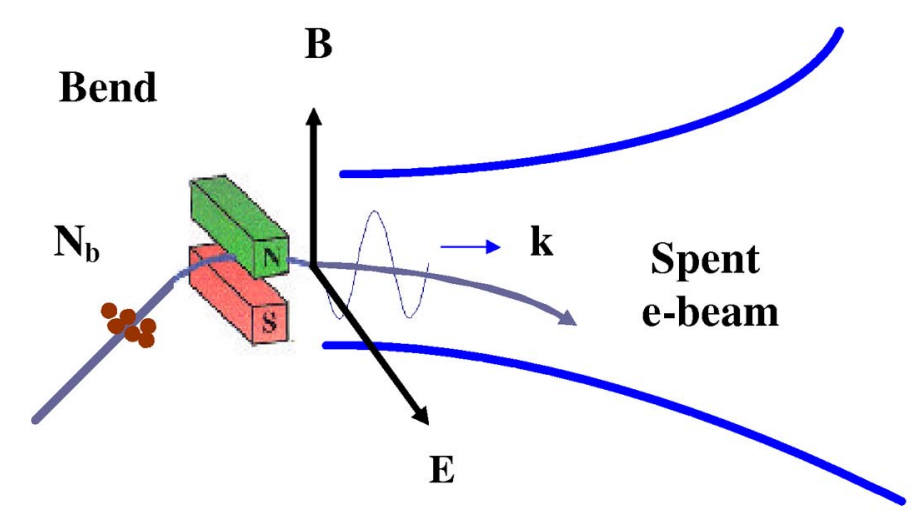

FIG. 1. (Color) Prebunched $e$-beam radiation schemes: (a) prebunched free electron maser (FEM)/FEL (or synchrotron-undulator radiation) and (b) coherent synchrotron radiation. 

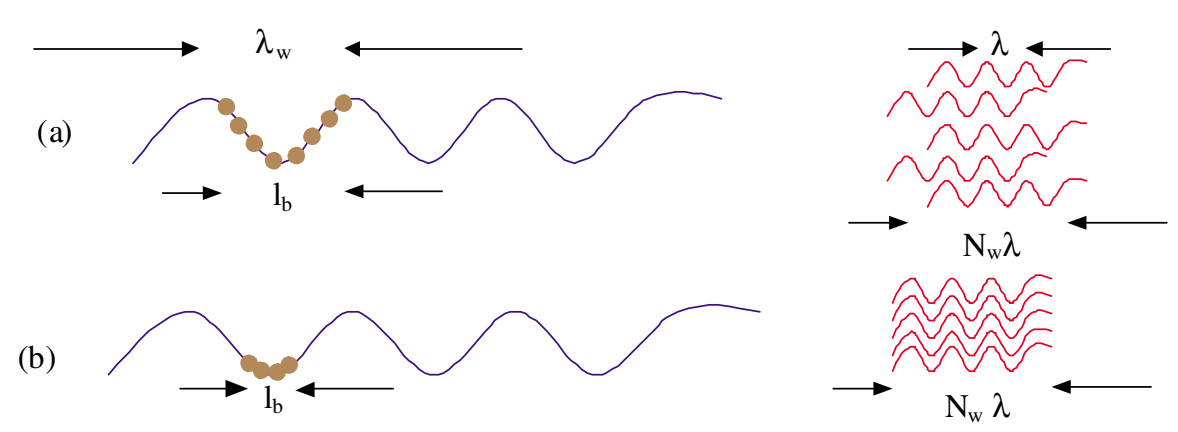

FIG. 2. (Color) Synchrotron-undulator emission from an electron beam bunch: (a) incoherent emission of uncorrelated radiation wave packets (FEL spontaneous emission) $l_{b}>\lambda$ and (b) coherent emission of phase correlated wave packets (superradiant emission) $l_{b} \ll \lambda$.

where $t_{b}$ is the electron bunch duration. If the electron beam moves near the speed of light, this condition can be written $l_{b} \ll \lambda$, where $l_{b}=t_{b} c$ is the bunch length.

Consider, for example, the case of a prebunched beam FEL or "synchrotron-undulator radiation" [Fig. 1(a)], in which the $e$-beam phase-space parameters satisfy the condition (1) for interaction with a single (free-space or waveguide) transverse mode at frequency $\omega$. Each electron will emit in free space into the radiation mode a radiation wave packet of duration $t_{s}=N_{w} \cdot 2 \pi / \omega$, where $N_{w}$ is the number of undulator periods (or, more generally, the number of oscillations performed by the electrons). All of the wave packets are emitted and propagate in phase, constituting a superradiant emission process [see Fig. 2(b)]. The resultant superposition field of the wave packets, emitted by the electron bunch, is proportional to the number of electrons in the bunch $N_{b}$, and therefore the radiated energy and instantaneous power are proportional to $N_{b}^{2}$. When condition (2) is not satisfied, as is the case in conventional synchrotron-undulator radiation [Fig. 2(a)], the wave packets interfere randomly. Their field average over all wave packets vanishes, and only their energies sum up in proportion to the number of electrons in the bunch $N_{b}$. Consequently, the radiated energy and instantaneous power, emitted by the single bunch in the superradiance limit (2), is significantly larger (factor $N_{b}$ ) than in common spontaneous undulator-synchrotron radiation. Apart from this difference, the radiation pattern and the radiation bandwidth $\left(\Delta \omega=2 \pi / t_{s} ; \Delta \omega / \omega \cong 1 / N_{w}\right)$ are the same in both cases.

If instead of a single bunch of $N_{b}$ electrons a large number of short bunches (a "macropulse") are injected into the undulator, the total radiative energy emitted into a radiation mode $q$ will grow in proportion to the number of bunches in the macropulse, $N_{M}$. If the bunches satisfy (2) and are injected periodically at a bunching frequency $\omega_{b}$ within the spectral bandwidth of the radiation process, then the field amplitudes of the wave packets, emitted by the individual bunches, will interfere (superimpose) in phase and thus the bandwidth of the emitted radiation reduces in inverse proportion to the macropulse duration (if $N_{M} \gg$
$N_{w}$, then $\left.\Delta \omega / \omega=1 / N_{M}\right)$. Consequently, the emission spectral energy $d W_{q} / d \omega$ will also grow in proportion to $N^{2}=N_{M}^{2} N_{b}^{2}$, where $N$ is the total number of electrons in the macropulse. At the same time, the power $P_{q}$ within the macropulse $\left(W_{q}\right.$ divided by the pulse duration) is, of course, independent of $N_{M}$, but the spectral power grows in proportion to $N_{M}$. In the limit of a continuously bunched electron beam $\left(N_{M}=\infty\right)$, the spectral power $d P_{q} / d \omega$ is theoretically infinite, and is not a relevant parameter. This is because the emitted radiation is then completely coherent, and its frequency bandwidth is zero. The scaling laws of the optical parameters of the radiation emitted into a single radiation mode by undulating $e$-beam radiators are summarized in Table I.

This general description and classification of bunched and unbunched electron-beam radiators is valid not only for undulator-synchrotron radiation, but for a wide class of electron-beam radiation schemes like electron cyclotron radiation, Cerenkov radiation, Smith-Purcell radiation, transition radiation, and more. A unified analysis of radiation from periodically bunched electron beams in this general class of electron-beam radiation schemes was presented by Schnitzer and Gover [4] for the small signal (linear) regime. Single bunch synchrotron-undulator radiation was analyzed by numerous contributors [5-8]. Both periodic and single bunch undulator-synchrotron radiators (prebunched beam FELs) were studied experimentally [9-13], demonstrating, respectively, the square law dependence of the radiation on the bunching current $I_{b}^{2}$ or bunch

TABLE I. Scaling of the optical parameters of the radiation into a single transverse mode by prebunched $e$-beam radiators.

\begin{tabular}{lccc}
\hline \hline Parameter & No bunching & Single bunch & Periodic bunching \\
\hline$\Delta \omega$ & $1 / N_{w}$ & $1 / N_{w}$ & $1 / N_{M}$ \\
$W_{q}$ & $N$ & $N_{b}^{2}$ & $N_{M} N_{b}^{2}$ \\
$d W_{q} / d \omega$ & $N$ & $N_{b}^{2}$ & $N_{M}^{2} N_{b}^{2}$ \\
$P_{q}$ & $N$ & $N_{b}^{2}$ & $N_{b}^{2}$ \\
$d P_{q} / d \omega$ & $N$ & $N_{b}^{2}$ & $N_{M} N_{b}^{2}$ \\
\hline \hline
\end{tabular}


electrons number $N_{b}^{2}$, as expected in superradiance processes. Similar observations were also reported with Cerenkov radiation schemes [19,30].

A concept closely related to the scheme of prebunched beam synchrotron-undulator radiation or prebunched beam FEL is the coherent synchrotron radiation; see Fig. 1(b). This radiation source has received great attention recently since it was used to demonstrate radiative emission in the $\mathrm{THz}$ frequency range at power levels, many orders of magnitude larger than formerly achieved [1].

As seen from Fig. 1(b), the CSR radiation source can be viewed as a PB-FEL of a single oscillation period $\left(N_{w}=1\right)$. If narrow bandwidth radiation is desirable, clearly the PB-FEL is advantageous and also produces higher power (energy), which grows in proportion to $N_{w}^{2}$. However, in some applications of radiation sources, rather a wide bandwidth is desirable. Consequently, CSR radiation sources [and coherent edge radiation (CER)] are presently of considerable interest. In this paper I will treat them with the same formulation, so that the relation between CSR and PB-FEL and other $e$-beam radiation devices can be easily quantified by direct application of the formulation.

Before proceeding, a note is in order concerning the techniques of electron bunching and their parameters. In accelerator technology, bunching of electrons emitted from thermionic guns is done by means of a powerful rf field in klystron cavities or acceleration sections, and it can be enhanced by use of a dispersive magnet (chicane). The recent development of photocathode electron guns, prompted by the impressive progress of high power picosecond and subpicosecond solid state lasers and nonlinear optical frequency multiplication techniques, brought about an abundance of accelerators operating with intense picosecond-pulsed electron beams [1]. Thus, according to (2), single bunch superradiance can be attained at frequencies as high as the terahertz regime. Continuous bunching at such high frequencies is not available yet, mostly because the solid state lasers cannot be modlocked and operated at such high repetition rates. However, various concepts have been suggested for attaining high frequency bunching, and recently Neumann et al. demonstrated generation of a finite train of electron microbunches (four) at $\mathrm{THz}$ frequency, using a comb spatial filter inside the cavity of the solid state laser [31]. This demonstration may lead to future development of various schemes of periodically bunched superradiant $e$-beam radiation sources in the THz regime.

\section{THEORETICAL FORMULATION}

It is convenient to solve the problem of radiation emission from $e$-beam radiation devices in the frequency domain. I use a formulation of modal expansion, where the traveling wave spectral radiation fields are expanded in terms of a complete set of transverse modes [32]:

$$
\begin{aligned}
\mathbf{E}(\mathbf{r}, \omega) & =\sum_{ \pm q} C_{q}(z, \omega) \tilde{\mathbf{E}}_{q}(\mathbf{r}), \\
\mathbf{H}(\mathbf{r}, \omega) & =\sum_{ \pm q} C_{q}(z, \omega) \tilde{\mathbf{H}}_{q}(\mathbf{r}),
\end{aligned}
$$

where $\{\underline{E}(\underline{\mathbf{r}}, \omega), \underline{H}(\underline{\mathbf{r}}, \omega)\}$ are the Fourier transforms of the corresponding time-domain fields, $\left\{\tilde{E}_{q}(\mathbf{r}), \tilde{H}_{q}(\mathbf{r})\right\}$ is a set of directed (propagating in the $+z$ or $-z$ direction) orthogonal modes normalized to an arbitrary radiation power $\mathrm{P}_{q}$,

$$
\frac{1}{2} \operatorname{Re} \iint \tilde{\mathbf{E}}_{q \pm} X \tilde{\mathbf{H}}_{q \pm} \cdot \hat{e}_{z} d A= \pm \mathrm{P}_{q}
$$

and $C_{q}(z, w)$ is the slowly growing field amplitude of the radiation mode $q$ at spectral frequency $\omega$ interacting with an electron beam along its propagation ( $z$ ) direction.

In many practical cases (especially at long wavelengths) I may choose the radiation modes to be real waveguide modes. In free space, one may choose as an expansion basis any set of optical modes in the Fresnel approximation (e.g., Hermit-Gaussian modes or Laguere-Gaussian modes). Furthermore, in free space one may also use expansion in terms of continuous radiation modes [32] (e.g., plane waves). In this case the mode parameter $q$ becomes continuous and the summation is replaced by integration. For example, if the expansion modes are plane waves, then

$$
\tilde{\mathbf{E}}_{q}(r)=\tilde{\mathbf{E}}_{\sigma \mathbf{k} \perp} e^{i \mathbf{k} \cdot \mathbf{r}},
$$

where $q=\left[\sigma, \mathbf{k}_{\perp}\right], \sigma$ is a polarization index, and $\mathbf{k}_{\perp}$ is the transverse component of the plane-wave wave number $\mathbf{k}$. In this case, the summation (3) turns into integration over the continuous transverse wave numbers $\mathbf{k}_{\perp}$, which is nothing but an inverse spatial Fourier transform of the plane wave spectral coefficients $C_{\sigma}\left(\mathbf{k}_{\perp}, z, \omega\right)$. Though the subsequent formulation can be extended to include (in case of free-space propagation) expansion in terms of continuous transverse modes (plane-wave expansion), for the most part of this and the subsequent paper I will use, not only in waveguides but also in cases of free-space propagation, a notation of discrete modes (specifically Hermit-Gaussian modes). This helps to treat in a unified way both free-space and waveguide radiation schemes, and provides useful optical characterization and simple figureof-merit parameters that quantify the coherent spectral radiation emission in all cases.

Utilizing the Lorentz theorem for the frequency domain Maxwell equations, driven by a current source $\mathbf{J}(\mathbf{r}, \omega)=$ $\int_{-\infty}^{\infty} \underline{J}(r, t) \exp (i \omega t) d t$, results in the following simple excitation equation for the radiation mode amplitudes [32]

$$
\frac{d C_{q}(z, \omega)}{d z}=-\frac{1}{4 \mathrm{P}_{q}} \int \mathbf{J}(\mathbf{r}, \omega) \cdot \tilde{\mathbf{E}}_{q}^{*}(\mathbf{r}) d A,
$$


which can be formally integrated

$$
C_{q}^{\text {out }}(\omega)-C_{q}^{\text {in }}(\omega)=-\frac{1}{4 \mathrm{P}_{q}} \int \mathbf{J}(\mathbf{r}, \omega) \cdot \tilde{\mathbf{E}}_{q}^{*}(\mathbf{r}) d V .
$$

For a current source composed of a finite number $(N)$ of electrons

$$
\mathbf{J}(\mathbf{r}, t)=\sum_{j=1}^{N}-e \mathbf{v}_{j}(t) \delta\left(\mathbf{r}-\mathbf{r}_{j}(t)\right) .
$$

This results in

$$
C_{q}^{\text {out }}(\omega)-C_{q}^{\text {in }}(\omega)=-\frac{1}{4 \mathrm{P}_{q}} \sum_{j=1}^{N} \Delta \mathrm{W}_{q j},
$$

where

$$
\Delta \mathrm{W}_{q j}=-e \int_{-\infty}^{\infty} \mathbf{v}_{j}(t) \cdot \tilde{\mathbf{E}}_{q}^{*}\left(\mathbf{r}_{j}(t)\right) e^{i \omega t} d t
$$

is a "complex spectral work function" related to the work done by electron $j$, when interacting with a normalized radiation mode $q$ along its path trajectory $\mathbf{r}_{j}(t)$.

If $\mathbf{r}_{j}(t), \mathbf{v}_{j}(t)$ can be calculated in the interaction region from the electron dynamical equations for all electrons, then $C_{q}^{\text {out }}(\omega)$ is found from (9) and (10), and subsequently the total spectral fields can be found by substitution of the mode amplitude in the modal expansion (3), and the real (time-domain) fields would be found by applying an inverse Fourier transformation on (3). However, this is usually not a preferred option, because of the large amount of computations involved, and mainly because the full mapping of the radiation field is often less useful to the user than radiometric optical parameters that characterize the coherent radiation source.

The spectral optical parameters can be calculated from the mode amplitudes using

$$
\begin{gathered}
\frac{d W_{r}}{d \omega}=\sum_{q} \frac{d W_{q}}{d \omega}, \\
\frac{d W_{q}}{d \omega}=\frac{2}{\pi} \mathrm{P}_{q}\left|C_{q}^{\text {out }}(\omega)\right|^{2} \\
=\frac{2}{\pi} \mathrm{P}_{q}\left|C_{q}^{\text {in }}(\omega)-\frac{1}{4 \mathrm{P}_{q}} \sum_{j=1}^{N} \mathrm{~W}_{q j}\right|^{2} .
\end{gathered}
$$

Here $d W_{q} / d \omega$ is defined for positive frequencies only, so that the total energy emission per mode can be found simply by integration over all frequencies:

$$
W_{q}=\int_{0}^{\infty} \frac{d W_{q}}{d \omega} d \omega
$$

This formulation is pretty general, and can be applied to all kinds of spontaneous, superradiant and stimulated electron-beam radiation devices, whether the beam is prebunched or not.
The complex spectral work function $\Delta \mathrm{W}_{q j}(\omega)$ is the only parameter that characterizes the specific radiation device or mechanism, and sets an upper bound on the radiation bandwidth. It can be written in general as

$$
\Delta \mathrm{W}_{q j}=\Delta \mathrm{W}_{q j}^{(0)}+\Delta \mathrm{W}_{q j}^{s t},
$$

where $\Delta \mathrm{W}_{q i}^{(0)}$ is the complex work function calculated with the zero order (in terms of the radiation field) electron trajectories $\mathbf{r}_{j}^{(0)}(t), \mathbf{v}_{j}^{(0)}(t)$ (the trajectories in the absence of radiation field):

$$
\Delta \mathbf{W}_{q j}^{(0)}=-e \int_{-\infty}^{\infty} \mathbf{v}_{j}^{(0)}(t) \cdot \tilde{\mathbf{E}}_{q}^{*}\left(\mathbf{r}_{j}^{(0)}(t)\right) e^{i \omega t} d t .
$$

This parameter is generally sufficient for calculating the spontaneous and superradiance emission from any $e$-beam radiation device. The second term in (14) $\Delta \mathrm{W}_{q j}^{s t}$ is the modification to the complex work function due to the presence of the field of the radiation mode $q$, and therefore it is the stimulated emission (or absorption) term. Its calculation requires solution of the electron force equation (to first order in the field amplitude $C_{q}$ in the linear-gain regime). The calculation of the stimulated emission term for specific radiation devices, e.g., FEL, is the subject of numerous publications. In the context of this paper I present this term just for the sake of completion and it will not be further developed. It is only pointed out that in the linear regime $\Delta \mathrm{W}_{q j}^{s t}$ is proportional to the mode field amplitude $C_{q}$, and therefore the stimulated emission field spectral component of the radiation wave packet generated by each electron $j$ in (9): $\Delta C_{q j}^{s t}=-\Delta \mathrm{W}_{q j}^{s t} / 4 \mathrm{P}_{q}$, is in phase with $C_{q}$ for all electrons $j$. This is displayed graphically in the complex mode-amplitude plane in Fig. 6(c).

\section{A. Optical characterization of a coherent radiation beam}

The use of a radiation beam, generated by a radiation source, requires, in general, optimizing the transport of the radiation beam by means of optical components, namely proper optical focusing and apperturing of the beam, in order to couple it onto the work or study sample. In the optical system design, one usually requires maximal radiative power or energy illumination of the user's target plane within certain area and ray-angle bounds. This four-dimensional $\left(\mathbf{r}_{\perp}, \mathbf{\Omega}\right)$ phase-space acceptance volume, defined by the application and the transmission optical system, determines the corresponding phase-space volume on the source, from which the radiation rays, arriving the target phase-space acceptance volume, emanate. By Liouville's theorem the magnitude of these volumes are equal in a lossless system. In principle, in conventional incoherent ray optics, one can quantify the radiation energy or power incident on the target, as the product of this conservative phase-space volume and the 
brightness field of the source $d W_{s}\left(\mathbf{r}_{\perp}, \boldsymbol{\Omega}\right) d^{2} r_{\perp} d \boldsymbol{\Omega}$ [or $\left.d P_{s}\left(\mathbf{r}_{\perp}, \mathbf{\Omega}\right) / d^{2} r_{\perp} d \Omega\right]$.

Figure 3(a) depicts the configuration of measuring the brightness of a source by two apertures. It also describes a typical application of delivery of incoherent radiation onto a target plane. If one desires to maximize the radiative energy or power delivered into a given area $\left(2 \Delta x_{t}\right)\left(2 \Delta y_{t}\right)$ in the target plane (maximum irradiance), the angular aperture $2 \Delta \theta_{s}$ should be increased to include most of the angular spread of the radiation lobe (in synchrotron and undulator radiation $\sim 2 / \gamma$ ).

Many applications of radiation require temporal and/or spatial coherence. Spectroscopic applications require temporal coherence. Applications in high resolution imaging, holography, microscopy, and, in general, attaining the smallest size (diffraction limited) spot, require spatial coherence. As shown in Fig. 3(a), temporal coherence, can be obtained by incorporating an optical filter in the beam path or, in practice, using a monochromator at the user station. Similarly, spatial coherence may be obtained by spatial filtering, namely, by setting optical apertures dimensions, that assure that the beam spot on target occupies phasespace volume of $\pi \Delta x_{t} \Delta \Theta_{t}<\lambda / 2=\pi \Delta x_{s} \Delta \Theta_{s}$.

Naturally, if the source is not coherent by itself, this filtering process will reduce substantially the available coherent radiative energy (power) on the target. This is the case, for example, with conventional synchrotron and undulator radiation sources in their domain of operation (UV, x ray). At these wavelengths, even after optical (frequency) filtering, the radiation is still spatially incoherent because the beam emittance does not satisfy condition (1). Spatial filtering is still possible in principle, but it would render significant attenuation of the radiation power.

When considering superradiant electron beam sources, the temporal condition (2) should be satisfied, and this necessarily corresponds to operation at long wavelengths ( $\mathrm{THz}$ regime). Condition (1) is then usually easily satisfied, and the radiation beam, at fixed frequency, is spatially coherent. It should be noted, though, that $e$-beam radiation sources of interest (prebunched FEL, CSR) are not point sources, but they are usually close to be longitudinal line sources [Fig. 3(b)]. Consequently, though their phase space

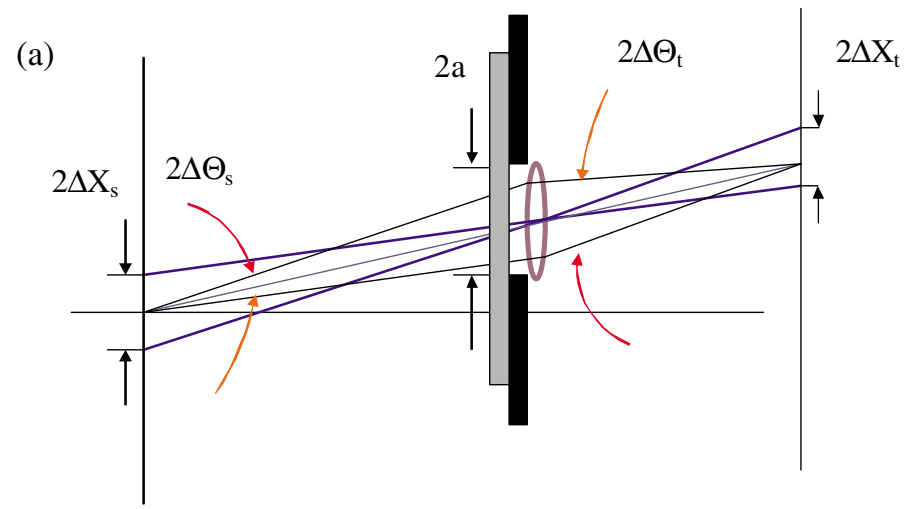

Planar Radiation - Source

Target Plane

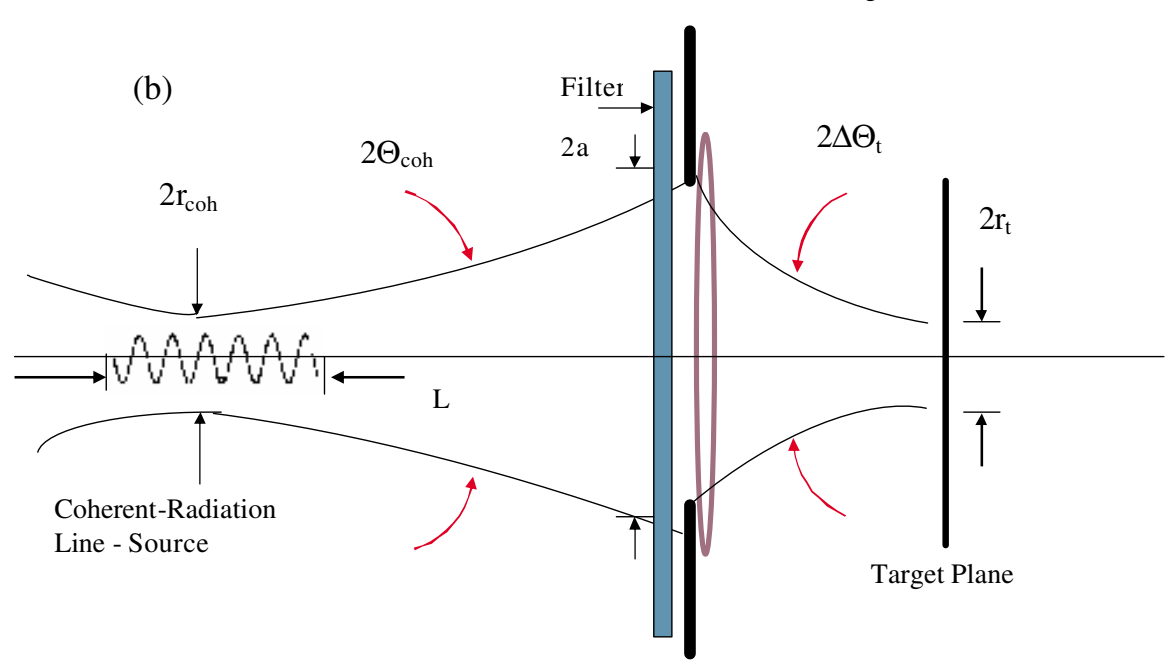

FIG. 3. (Color) Schematic (single thin lens) appertured optical delivery system from a radiation source to a target. (a) Spatial filtering and brightness measurement of an incoherent radiation source. (b) Effective spatial and angular apertures of a spatially coherent line radiation source. 
area is $\int x d \Theta_{x}=\lambda / 2$, their phase-space distribution is quite irregular (see the discussion in the subsequent paper), and if transmitted through a diffraction limited aperture $\pi \Delta x \Delta \Theta=\lambda / 2$, as shown in Fig. 3(b), a significant part of the (filtered) spatially coherent radiation energy (power) would be cutt off. Alternatively stated, if the radiation beam would have been focused by a nonappertured lens large enough to include the entire radiation lobe, its focal spot would be larger than an ideal diffraction limited spot attainable from a plane wave incident on the same aperture. Therefore, even though the line source is theoretically spatially coherent, its "useful" spatially coherent power (or energy) would be only the fraction that can pass through a spatial filter satisfying $\pi \Delta x \Delta \Theta=\lambda / 2$.

From this discussion it turns out that the "useful coherent spectral energy (or power)" of the source, namely the energy (power) per unit frequency, that can be delivered to the work with diffraction limited optics, is the relevant figure of merit for characterizing coherent radiation sources of different kinds and comparing them quantitatively (including comparison between superradiance and spontaneous emission processes that is useful, for example, in order to determine the signal-to-noise ratio of the same radiation source). It is proposed, therefore, that a good figure of merit for estimating useful coherent spectral energy (power) emitted in free space by a source is the energy (power) excited in the free-space Gaussian wave

$$
\begin{aligned}
\tilde{\mathbf{E}}_{q} & =\tilde{\mathbf{E}}_{00} \\
& =\frac{w_{0}}{w(z)} \exp \left[-\frac{x^{2}+y^{2}}{w^{2}(z)}-i k \frac{x^{2}+y^{2}}{2 R(z)}+i \varphi+i k z\right]
\end{aligned}
$$

which is the fundamental transverse mode in the expansion for the fields (3) in terms of the Hermit-Gauss free-space transverse modes [33]. This mode, common in laser design, couples efficiently through the circular apertures of regular lenses and mirrors. This concept of using a Gaussian mode to modelize the coherent radiation from an extend line source, such as an undulating electron, has been used before to calculate the brightness of synchrotron-undulator radiation emission [34].

In the Gaussian wave model, the radiation beam-spot radius is $w(z)=w_{0} \sqrt{1+z^{2} / z_{R}^{2}}$, where $w_{0}$ is the beam waist spot size and $z_{R}=\pi w_{0}^{2} / \lambda$ is the Rayleigh diffraction length. Thus, the diffraction half-cone angle of the Gaussian beam is

$$
\Theta_{\text {diff }}=\frac{1}{\pi} \frac{\lambda}{w_{0}} .
$$

The Gaussian wave waist spot size $w_{0}$, waist position $(z=$ 0 ), and propagation direction ( $z$ axis), that should be used in the calculation of the useful spatially coherent energy (power), are free parameters. The reasonable choice is the one that provides maximum collection of radiation from the electron beam and maximum transmission through the radiation-collection optics and apertures. Optimal radiation collection optics will define a Gaussian beam that maximizes the weighed work function (15) of the electrons in the $e$-beam.

In the free-space radiation-emission schemes, analyzed in this paper, the radiation emission is always centered around the electron beam propagation direction ( $z$ axis). The effective waist size $w_{0}$ (closely related to the source coherence width) will be defined so that uniform interaction (negligible beam diffraction) takes place along the entire length $L$, namely the beam waist is chosen in the center of the interaction region and its Rayleigh length satisfies

$$
L=2 z_{R}=2 \pi w_{0}^{2} / \lambda .
$$

From (17) and (18) the diffraction angle of the radiation beam of an extended line source is

$$
\Theta_{\mathrm{diff}}=\frac{2^{3 / 2}}{\sqrt{\pi}} \sqrt{\frac{\lambda}{L}} .
$$

The concept of useful spatially coherent spectral energy (power) introduced here has limited validity. Calculation of the radiative energy emission into the Gaussian mode (16) with waist size $w_{0}$ defined by (18) gives a good estimate of the useful coherent radiative energy only if the emission is in free space and the optical transmission optics is matched to collect optimally this radiation mode, and delivers it without loss onto the target. Also, the choice of the Gaussian waist size (18) is valid only if the radiating electron can keep in phase along the distance $L$ with a free-space plane wave that it emits in the $z$ direction. In all other cases one needs to include the contributions of all the higher order free-space radiation modes to the sum (11), and then include in the calculation the radiative power reduction due to the diffraction and aperture interception in the transmission optics beyond the radiation source. The use of a single fundamental Gaussian mode (16) in the calculation of the "useful spectral energy" (12) and (15) is also valid in the case that the delivery optics is diffraction limited. In this case the mode waist size and waist position are defined by the optics [not by (18)], and the calculation of (12), (9), and (15) gives the spectral radiative energy collected by this optical system (which may be smaller than the maximal useful coherent radiative energy attainable in a more optimal optical system).

\section{B. Single electron emission in an undulator}

The zero order complex work function $\Delta \mathrm{W}_{q j}^{(0)}$ (15) can be calculated straightforwardly for any given radiation device. To reduce the analysis from the abstract to the concrete I calculate it now for two examples of interest: first for undulator radiation and, in the next section, for synchrotron radiation from a bending magnet. Now assume 
an accelerated electron performing periodic harmonic motion along axis $z$,

$$
\mathbf{v}_{j}(t)=\operatorname{Re}\left(\tilde{\mathbf{v}}_{\perp j} e^{-i k_{w} z_{j}(t)}\right)+v_{z} \hat{e}_{z},
$$

which is the case for undulator radiation or FEL (but also cyclotron resonance maser). In an undulator $\tilde{\mathbf{v}}_{\perp j}=$ $e \mathbf{B}_{w} / \gamma_{j} m k_{w}$, where $\tilde{\mathbf{B}}_{w}$ is the complex amplitude of the periodic transverse magnetic field.

Assume that the electron beam is narrow enough so that all electrons experience the same field when interacting with the mode [in free-space interaction, Eq. (1) must be satisfied as a necessary condition]:

$$
\tilde{\mathbf{E}}_{q}\left(\mathbf{r}_{j}^{(0)}(t)\right)=\mathbf{E}_{q}\left(\mathbf{r}_{\perp 0}\right) e^{i k_{z q} z_{j}^{(0)}(t)},
$$

where $z_{j}^{o}(t)=v_{z}\left(t-t_{o j}\right)$, and $\mathbf{r}_{\perp 0}$ is the transversecoordinates vector of the electron beam. Substituting this and (20) in (15) one obtains

$$
\Delta \mathrm{W}_{q j}^{(0)}=-e \frac{\mathbf{v}_{\perp 0} \cdot \mathbf{E}_{q}^{*}}{2 v_{z}} L \sin c(\theta L / 2) e^{i \theta L / 2} e^{i \omega t_{o j}},
$$

where $L=N_{w} \lambda_{w}$ is the interaction length $\left(\lambda_{w}=2 \pi / k_{w}\right)$, $\sin c(x) \equiv \sin x / x$, and $\theta(\omega)$, the detuning parameter, is defined by

$$
\theta(\omega)=\frac{\omega}{v_{z}}-k_{z q}(\omega)-k_{w} .
$$

The detuning function $\sin c(\theta L / 2)$ attains its maximum value at the synchronism frequency $\omega_{o}$ defined by

$$
\theta\left(\omega_{0}\right)=\frac{\omega_{0}}{v_{z}}-k_{z q}\left(\omega_{0}\right)-k_{w}=0
$$

Near synchronism

$$
\theta(\omega) L \cong\left(\omega-\omega_{0}\right) t_{s} \equiv 2 \pi \frac{\omega-\omega_{0}}{\Delta \omega},
$$

where

$$
t_{s}=\frac{2 \pi}{\Delta \omega}=\frac{L}{v_{z}}-\frac{L}{v_{g q}}
$$

is the wave packet slippage time and $v_{g q} \equiv d \omega /\left.d k_{z q}\right|_{\omega_{0}}$ is the group velocity of the mode. The spectral energy lineshape function $\sin c^{2}(\theta L / 2)$ is drawn in Fig. 4.

In free space $k_{z q}=\omega / c, v_{g q}=c$, and the solution of (24) is

$$
\omega_{0}=\frac{c k_{w}}{1 / \beta_{z}-1} \cong 2 \gamma_{z}^{2} c k_{w}=\frac{2 \gamma^{2}}{1+a_{w}^{2} / 2} c k_{w},
$$

where $a_{w}=\gamma v_{\perp 0} / c=e B_{w} /\left(k_{w} m c\right)$. The second part of Eq. (27) is valid only for a linearly polarized wiggler in the highly relativistic limit $\beta_{z} \rightarrow 1$. The spectral bandwidth then is

$$
\Delta \omega=\frac{\omega_{0}}{N_{w}}
$$

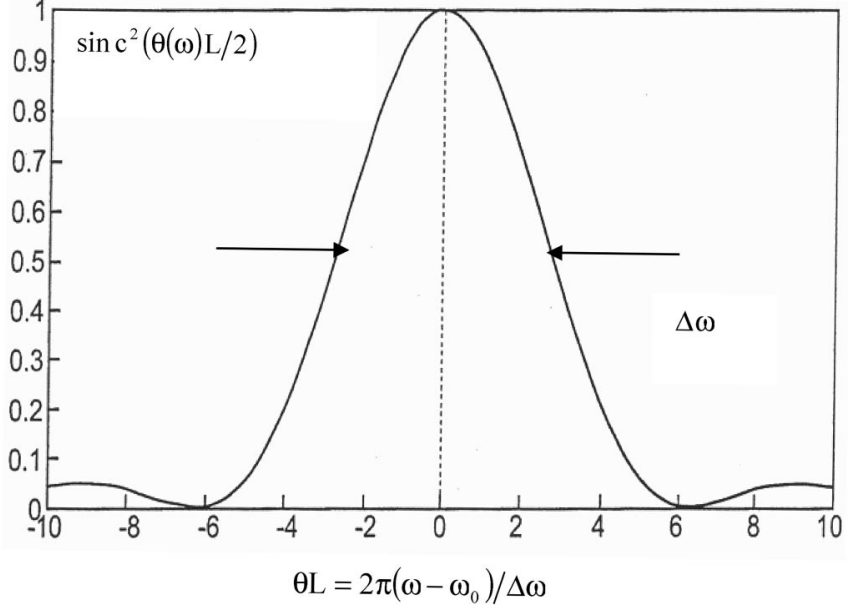

FIG. 4. The spectral energy line-shape function of radiation from an oscillating electron emitting a wave packet of finite duration $t_{s}$. For PB-FEL, see (23)-(28).

Clearly the undulator-synchrotron radiation spectral energy grows up in amplitude and narrows down in bandwidths as the undulator length $L$ (or number of wiggles $N_{w}$ ) grows (22) and (28).

\section{Single electron synchrotron emission in a magnetic bend}

The analysis of synchrotron radiation from a magnetic bend was carried out by numerous authors starting with Schwinger [35]. A detailed analysis of this problem, including the effect of prebunched beam CSR and its implications to bunch diagnostics, has been presented recently by Geloni et al. [36], based on a time-domain analysis.

In this section I derive, in the framework of the frequency-domain modal-expansion formulation, similarly to the undulator-radiation case, a simple expression for the "complex spectral energy" of an electron traversing a magnetic bend. This will be used later straightforwardly for calculating the spectral parameters of CSR.

Assume that the electron passes along a circular trajectory of radius $R_{e}$ within a uniform-field magnet section of intensity $B_{b}$ and length $L$. Within a paraxial approximation the electron trajectory and transverse velocity are given by

$$
\begin{gathered}
x_{j}(t)=\frac{1}{2 R_{e}} z_{j}^{2}(t), \quad\left[-\frac{L}{2}<z_{i}(t)<\frac{L}{2}\right], \\
z_{j}(t)=v\left(t-t_{0 j}\right), \\
v_{x j}(t)=\frac{v}{R_{e}} z_{j}(t),
\end{gathered}
$$

where

$$
R_{e}=\frac{\gamma m \beta c}{e B_{b}}
$$


Substituting (29)-(31) in (15), the integration along the electron trajectory $\underline{r}_{j}(t)$ can be performed quite simply, for any waveguide or free space mode of the form (21). If the electron can be assumed to make along this path only small displacement off axis, within which the transverse variation of the radiation mode is negligible, then

$$
\Delta \mathrm{W}_{q j}^{0}=i \frac{e}{R_{e}} \mathbf{E}_{q x}^{*} L^{2}\left[\frac{d}{d \theta L} \sin c(\theta L / 2)\right] e^{i \omega t_{o j}},
$$

where

$$
\theta=\frac{\omega}{v_{z}}-k_{z q}(\omega)
$$

and in general $k_{z q}=\frac{\omega}{c} \cos \Theta_{q}\left(\Theta_{q}\right.$ is the emission angle into a plane wave or a waveguide mode "zigzag" angle). In free-space forward emission [e.g., into a Gaussian mode (16)], $k_{z q}=\frac{\omega}{c}$ and

$$
\theta=\frac{\omega}{c}\left(\beta^{-1}-1\right) \approx \frac{1}{2 \gamma^{2}} \frac{\omega}{c},
$$

where the second part of the equation is valid for the limit $\gamma \gg 1$.

The spectral emission function $\left[\frac{d}{d \theta L} \sin c(\theta L / 2)\right]^{2}$ is shown in Fig. 5. This is a wideband emission function. Contrary to Fig. 4, it has no resonant frequency. It has a maximum "cutoff" frequency, which for free space propagation (35) is given by

$$
\omega_{c}=3 \pi\left(\beta^{-1}-1\right) \frac{c}{L} \cong 6 \pi \gamma^{2} \frac{c}{L},
$$

corresponding to $\theta_{c} \cong 3 \pi$, and it arrives to a maximum around

$$
\omega_{m}=2 \pi \gamma^{2} \frac{c}{L}
$$

corresponding to $\theta_{m} \cong \pi$.

Alternatively stated, constructive interference of coherent synchrotron radiation emission in the forward direction (z) by a single electron or a tight bunch of electrons is possible at long enough wavelengths: $\lambda>\lambda_{c}=2 \pi c / \omega_{c}$ (and attains maximal value at $\lambda=\lambda_{m}=\lambda_{c} / 3$ ), only for a limited propagation distance $L<L_{\mathrm{coh}}$, where $L_{\mathrm{coh}}$, the "coherence length" or "forming length," is given by

$$
L_{\text {coh }}=3 \gamma^{2} \lambda_{c}=\gamma^{2} \lambda_{m}
$$

Beyond $L_{\text {coh }}$ the radiation emission at this wavelength range gets out of phase with the radiation emitted beforehand.

This limitation is an absolute limitation due to the lack of "phase matching" between the radiating electron and the free-space propagating fast radiation wave. It cannot be circumvented by using a waveguide. In fact, in a waveguide [where in Eq. (34) $k_{z q}=\frac{\omega}{c} \cos \Theta_{q}$ with $\Theta_{q} \neq 0$ ] the coherence length becomes even shorter for a given frequency. Only the use of a dialectric medium or a slow wave

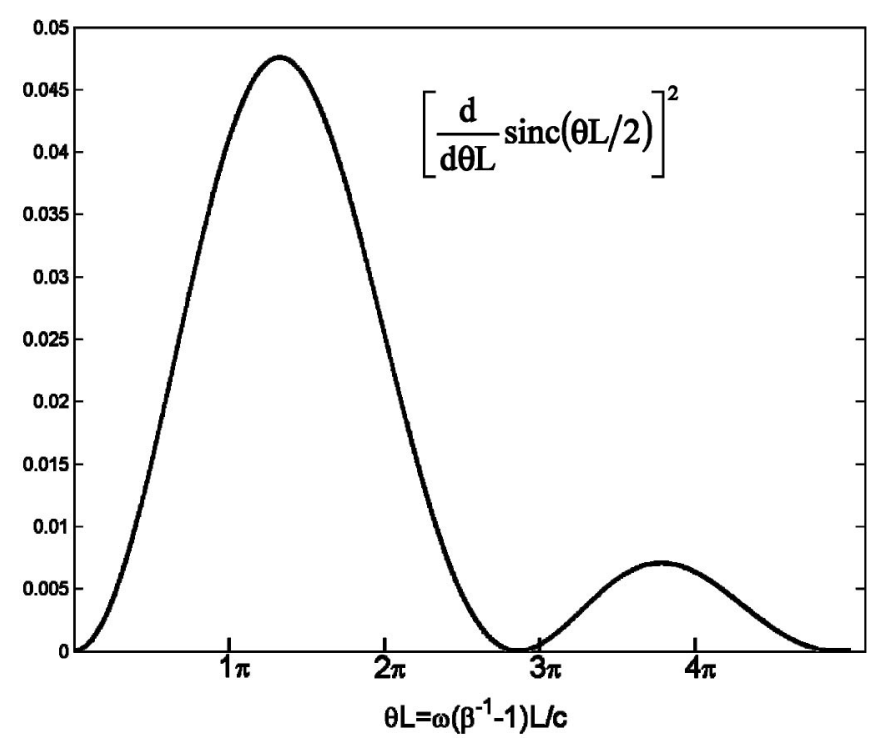

FIG. 5. The spectral energy line-shape function of the CSR radiation wave packet emitted by an electron passing through a magnetic bend (34).

structure can help to increase the coherence length and alleviate its limitation. In practice, for coherent synchrotron emission from high energy electron bunches in the $\mathrm{THz}$ regime, the coherence length can be quite longer than the bending magnet length $L$. For the Thomas Jefferson Laboratory (TJL) experiment [1] $\quad(\gamma=81, \lambda=$ $300 \mu \mathrm{m}, L=0.173 \mathrm{~m}$ ) one has $L_{\text {coh }}=2 m \gg L$.

A note is in order with respect to the validity of the new analytical expression (33) for the CSR "finite length broadened" spectral emission curve. Contrary to the PBFEL line-shape function (22), its validity is limited only to short enough interaction length (magnet length $L$ ). When one employs this expression for free-space CSR emission into a Gaussian wave (16) of given waist size $w_{0}$ and Rayleigh length $z_{R}=\pi w_{0}^{2} / \lambda$, the magnet length $L$ should be shorter than $2 z_{R}$, and the electron's displacement off the mode axis along the magnet length $L$ should not exceed its waist size:

$$
x_{j}( \pm L / 2)=\frac{L^{2}}{8 R_{e}}<w_{0},
$$

which is a stricter condition than the requirement $L<L_{\mathrm{coh}}$ (38).

Equation (33) can be used for for calculating the maximum useful spectral energy emitted into a Gaussian wave by setting $L \approx 2 z_{R}$ (18). However, this is valid only as an approximate estimate. For a more accurate estimate of this parameter, one must resort to numerical calculation, which would include in the contour integral of (15) the transverse field variation of the Gaussian mode (16), the electron phase variation due to the wave front curvature $R(z)$ and the Guoy phase $\varphi(z)$, and the accurate circular electron trajectories [instead of (29)-(31)]. This calculation is out of the scope of this paper [37]. 


\section{Emission from a single bunch}

Going back now to the general analysis for all $e$-beam radiation devices, I assume that the electron beam is narrow enough (1) so that all electrons arriving at different times $t_{j o}$ to a given reference plane $z_{\text {in }}=0$ in the interaction region go through the same trajectories in the interaction region: $\left\{\mathbf{r}_{j}^{(0)}(t), \mathbf{v}_{j}^{(0)}(t)\right\}=\left\{\mathbf{r}_{e}^{(0)}\left(t-t_{o j}\right), \mathbf{v}_{e}^{(0)}\left(t-t_{o j}\right)\right\}$. I note that, independently of the functional details of $\Delta \mathrm{W}_{q j}^{(0)}$ (namely independently of the radiation mechanism), it is possible, in general, to write (15) [similarly to the special cases of undulator radiation (22) and CSR radiation (33)] in the form

$$
\begin{gathered}
\Delta \mathrm{W}_{q j}^{(0)}=\Delta \mathrm{W}_{q e}^{(0)} e^{i \omega t_{j o}} \\
\Delta \mathrm{W}_{q e}^{(0)}=-e \int_{-\infty}^{\infty} \mathbf{v}_{e}^{(0)}(t) \cdot \tilde{\mathbf{E}}_{q}^{*}\left(\mathbf{r}_{e}^{(0)}(t)\right) e^{i \omega t} d t
\end{gathered}
$$

This is true of course only in the limit of a tightly narrow beam, in which all the electrons experience the same field of mode $q$ along their path through the interaction region. ${ }^{2}$ In the case of radiation in free space, the mode $q$ to be used is the fundamental Gaussian mode (16). Its parameters are defined by the optical system collecting the radiation or by Eq. (18).

Using (40) in (9) and in (12), I obtain

$$
\begin{aligned}
C_{q}^{\mathrm{out}}(\omega) & =C_{q}^{\mathrm{in}}(\omega)+\Delta C_{q e}^{(0)}(\omega) \sum_{j=1}^{N} e^{i \omega t_{o j}}+\sum_{j=1}^{N} \Delta C_{q j}^{s t} \\
& =C_{q}^{\mathrm{in}}(\omega)-\frac{1}{4 \mathrm{P}_{q}} \Delta \mathbf{W}_{q e}^{(0)} \sum_{j=1}^{N} e^{i \omega t_{0 j}}-\frac{1}{4 \mathrm{P}_{q}} \sum_{j=1}^{N} \Delta \mathbf{W}_{q j}^{s t},
\end{aligned}
$$

$$
\begin{aligned}
\frac{d W_{q}}{d \omega}= & \frac{2}{\pi} \mathrm{P}_{q}\left\{\left|C_{q}^{\mathrm{in}}(\omega)\right|^{2}+\left|\Delta C_{q e}^{(0)}(\omega) \cdot \sum_{j=1}^{N} e^{i \omega t_{o j}}\right|^{2}\right. \\
& +\left[C_{q}^{\mathrm{in} *}(\omega) \cdot \Delta C_{q e}^{(0)}(\omega) \cdot \sum_{j=1}^{N} e^{i \omega t_{o j}}+\text { c.c. }\right] \\
& \left.+\left[C_{q}^{\mathrm{in} *}(\omega) \cdot \sum_{j=1}^{N} \Delta C_{q j}^{s t}(\omega)+\text { c.c. }\right]\right\} \equiv\left(\frac{d W_{q}}{d \omega}\right)_{\mathrm{in}} \\
& +\left(\frac{d W_{q}}{d \omega}\right)_{\mathrm{sp} / \mathrm{SR}}+\left(\frac{d W_{q}}{d \omega}\right)_{\mathrm{ST}-\mathrm{SR}}+\left(\frac{d W_{q}}{d \omega}\right)_{s t}
\end{aligned}
$$

where in the last equation a term of second order in $\Delta C_{q}^{s t}$ was omitted (small gain limit).

\footnotetext{
${ }^{2}$ Note that if this narrow beam condition is not satisfied, the formulation (5)-(15) is still valid, and averaging over the ensemble of electron initial conditions will yield (due to spatial and angular $e$-beam spread) "inhomogeneous broadening" expressions for the spectral parameters. However, some of the general observations that I will make in the remainder of this section may be invalid in this inhomogeneous broadening limit.
}

The role of electron prebunching in effecting the radiation characteristics of any radiation scheme can now be lucidly explained with the aid of Fig. 6, showing the superposition in complex plane of the amplitudes of modal wave packets, emitted by the individual electrons. Figure 6(a) shows the process of spontaneous emission: superposition of wave packets, emitted by uncorrelated electrons at random phases [compare to Fig. 2(a)]. When the electrons are injected at random,

$$
\left\langle\left[\omega\left(t_{o j}-t_{0}\right)\right]^{2}\right\rangle^{1 / 2}>2 \pi
$$

the phase angle of the resultant amplitude $C_{q}^{\text {out }}$ is random, and therefore the spectral components of the amplitude of the radiation mode vanish on the average at all frequencies,

$$
\left\langle\left[\Delta C_{q}(\omega)\right]_{\mathrm{sp}}\right\rangle=0
$$

however, the absolute value of the amplitude is, on the average, finite. Assuming no correlation between the entrance phases of the electrons, the mixed terms in the squared sum in the second term of (43) vanish when $d W_{q} / d \omega$ is averaged over all phases: $\left\langle e^{i \omega\left(t_{o j}-t_{o j^{\prime}}\right)}\right\rangle=\delta_{j j^{\prime}}$. The second term then results in the expression for the spontaneous emission spectral energy:
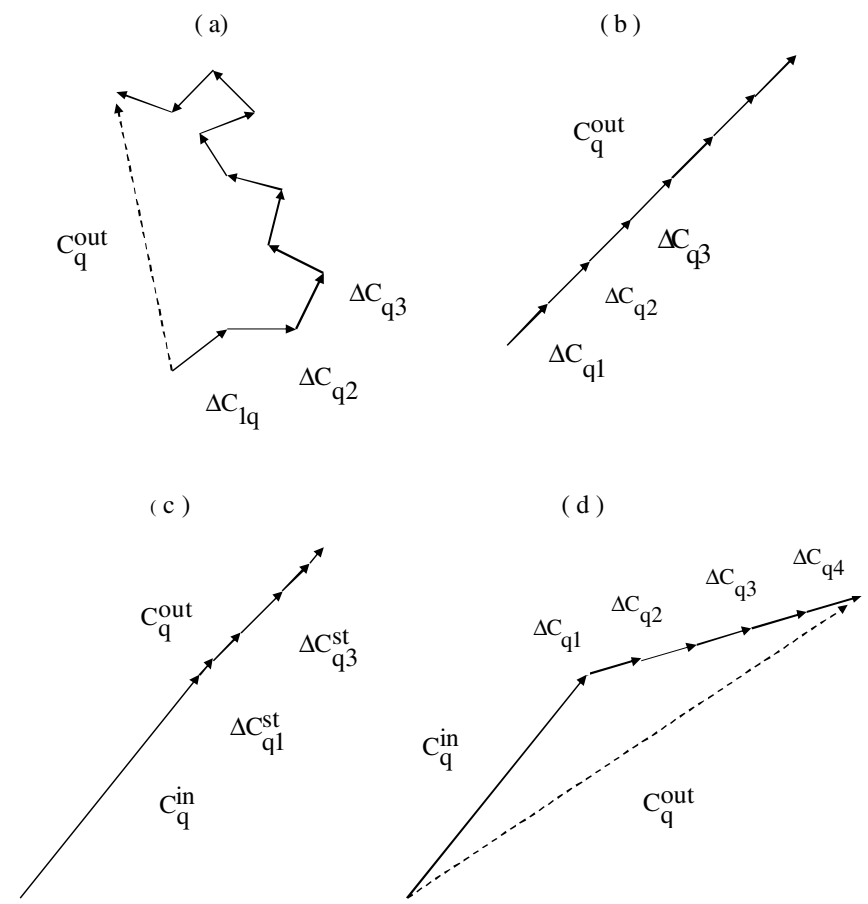

FIG. 6. Schematic description of the four radiative emission processes in the complex plane of the mode amplitude $C_{q}^{\text {out }}$, which is composed of $C_{q}^{\text {in }}$ and the superposition of the radiation wave packets $\Delta C_{q j}$ : (a) spontaneous emission, (b) superradiance, (c) stimulated emission, and (d) stimulated superradiance. 


$$
\left\langle\left(\frac{d W_{q}}{d \omega}\right)_{\mathrm{sp}}\right\rangle=\frac{2}{\pi} \mathrm{P}_{q}\left|\Delta C_{q}^{(0)}(\omega)\right|^{2} N=\frac{1}{8 \pi} \cdot \frac{\left|\Delta \mathrm{W}_{q e}^{0}\right|^{2}}{\mathrm{P}_{q}} N .
$$

Figure 6(b) shows the case of ideal superradiant emission from an electron beam bunch, in which all electrons have the same initial oscillation phase:

$$
\left\langle\left[\omega\left(t_{0 j}-t_{0}\right)\right]^{2}\right\rangle^{1 / 2} \ll 2 \pi .
$$

In this case one can substitute in (42) $\sum_{j=1}^{N} e^{i \omega t_{0 j}}=N e^{i \omega t_{0}}$, and consequently the second term in (43) results in the superradiance spectral energy:

$$
\left(\frac{d W_{q}}{d \omega}\right)_{\mathrm{SR}}=\frac{1}{8 \pi} \cdot \frac{\left|\Delta \mathrm{W}_{q e}^{(0)}\right|^{2}}{\mathrm{P}_{q}} N^{2}=\left\langle\left(\frac{d W_{q}}{d \omega}\right)_{\mathrm{sp}}\right\rangle N .
$$

Figure 6(d) displays a process that I propose to call stimulated superradiance: all electrons oscillate in phase, but because a radiation mode of distinct phase $\angle\left[C_{q}^{\text {in }}(\omega)\right]=\varphi_{q r}$ is injected in, the third term in (43) will contribute positive or negative energy, depending whether the electron bunch oscillates in phase or out of phase with the input radiation field. If the phase of the electron bunch relative to the wave is $\varphi=\omega t_{0}-\varphi_{q r}+$ $\angle\left(\Delta \mathrm{W}_{q e}^{(0)}\right)$, then the third term in (43) represents stimulated-superradiance spectral energy:

$$
\left(\frac{d W_{q}}{d \omega}\right)_{\mathrm{ST}-\mathrm{SR}}=-\frac{1}{2 \pi} \cdot\left|C_{q}^{\mathrm{in}}(\omega)\right|\left|\Delta \mathrm{W}_{q e}^{(0)}\right| N \cos \varphi .
$$

For the purpose of comparison I also display in Fig. 6(c) the process of conventional stimulated emission [fourth term in (43)]. In this process, the electron trajectories are modified by the radiation field of the mode within the interaction region. If $\Delta \mathbf{W}_{q j}^{s t}$ [Eq. (14)] is calculated to first order in the radiation field amplitude $C_{q}^{\text {in }}(\omega)$, then all the electrons, regardless of their initial phase, contribute to $\Delta \mathrm{W}_{q}^{s t}(\omega)$ terms, in phase (or counterphase) with $C_{q}^{\text {in }}(\omega)$. This would result in the expressions for stimulated emission (laser gain) or stimulated absorption from the $e$-beam. However the calculation of $\Delta \mathrm{W}_{q}^{s t}$ is out of the scope of this paper.

I note, in conclusion, that the conventional stimulated emission process exists whenever there is an input radiation field and the $e$-beam is unbunched; the spontaneousemission and superradiance processes take place (independently of having an input radiation field) when the $e$-beam is random or bunched, respectively; the new term of stimulated-superradiance process [third term in (43)] exists only when there are both input radiation field and electron prebunching simultaneously.

At this point I extend the analysis to include partial bunching, namely electron beam bunches of finite duration and arbitrary pulse-shape function. One can characterize the distribution of electron entrance times $t_{o j}$ of the elec- tron bunch by means of a normalized pulse-shape function $f\left(t_{0}^{\prime}-t_{0}\right)=i\left(t_{0}^{\prime}-t_{0}\right) /(e N)$, where $i(t)$ is the $e$-beam bunch current, and $t_{0}$ is bunch center entrance time:

$$
\int_{-\infty}^{\infty} f\left(t_{0}^{\prime}-t_{0}\right) d t_{0}^{\prime}=1
$$

Then the summation over $t_{o j}$ may be substituted by integration over entrance times $t_{0}^{\prime}$ :

$$
\begin{gathered}
\sum_{j=1}^{N} e^{i \omega t_{0 j}}=N \int f\left(t_{0}^{\prime}-t_{0}\right) e^{i \omega t} d t_{0}^{\prime}=N e^{i \omega t_{0}} M_{b}, \\
M_{b}(\omega)=\int_{-\infty}^{\infty} f\left(t_{0}^{\prime}\right) e^{i \omega t_{0}^{\prime}} d t_{0}^{\prime} .
\end{gathered}
$$

So, $M_{b}(\omega)$ is the bunching amplitude at frequency $\omega$. It modifies Eqs. (48) and (49) to

$$
\begin{gathered}
\left(\frac{d W_{q}}{d \omega}\right)_{\mathrm{SR}}=\frac{1}{8 \pi} \cdot \frac{\left|\Delta \mathrm{W}_{q e}^{(0)}\right|^{2}}{\mathrm{P}_{q}} M_{b}^{2} N^{2}, \\
\left(\frac{d W_{q}}{d \omega}\right)_{\mathrm{ST}-\mathrm{SR}}=-\frac{1}{2 \pi} \cdot\left|C_{q}^{\mathrm{in}}(\omega)\right| \cdot\left|\Delta \mathrm{W}_{q e}^{(0)}\right| M_{b} N \cos \varphi .
\end{gathered}
$$

In conditions of perfect bunching $f\left(t_{0}\right)=\delta\left(t_{0}\right)$, and consequently $M_{b}=1$, and Eqs. (48) and (49) are restored. For an example of a Gaussian electron beam bunch distribution $f\left(t_{0}\right)=\exp \left(-t_{0}^{2} / t_{b}^{2}\right) /\left(\sqrt{\pi} t_{b}\right)$, one obtains $M_{b}(\omega)=$ $\exp \left(-\omega^{2} t_{b}^{2} / 4\right)$. The bunching form factor $\left|M_{b}(\omega)\right|^{2}$ is drawn for this case in Fig. 7. Evidently, in the tight bunching condition (2) $\left|M_{b}\right|^{2} \cong 1$.

\section{E. Periodic bunching}

Assume that the beam consists of a finite train (macropulse) of electron bunches injected periodically at fre-

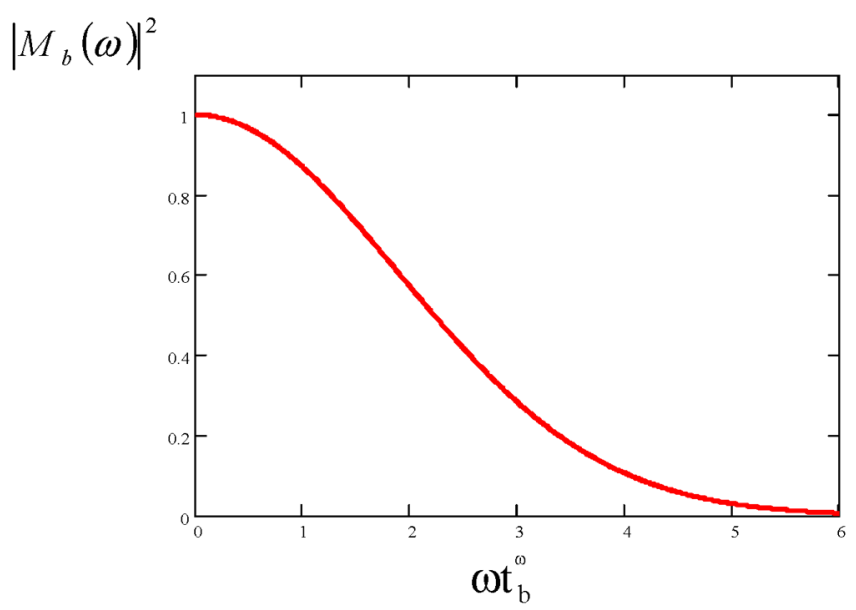

FIG. 7. (Color) The bunching form factor $\left|M_{b}(\omega)\right|^{2}=$ $\exp \left(-\omega^{2} t_{b}^{2} / 2\right)$ for a Gaussian $e$-beam bunch distribution $f\left(t_{0}\right)=$ $\exp \left(-t_{0}^{2} / t_{b}^{2}\right) /\left(\sqrt{\pi} t_{b}\right)$. 


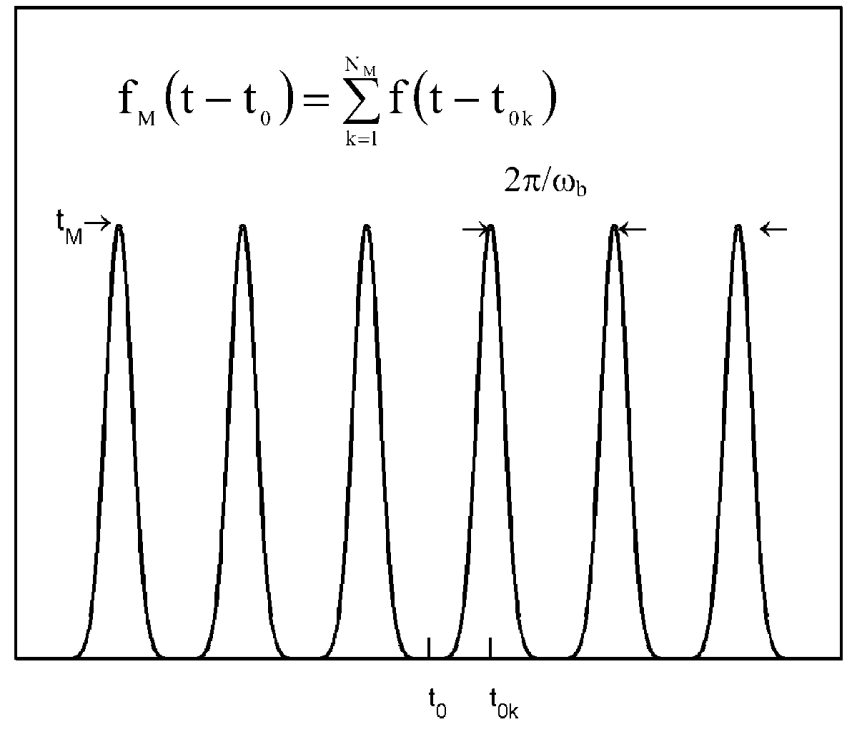

FIG. 8. The macropulse pulse-shape function, composed of a periodic train of $N_{M}$ microbunches at periodicity $2 \pi / \omega_{b}$.

quency $\omega_{b}$ into the interaction region (see Fig. 8). To take this into consideration requires to include summation over the $N_{M}$ bunches of the macropulse, when summing over all electrons in (42) and (43). Assuming a uniform train of electron bunches, in which all bunches have the same pulse-shape function $f\left(t_{0}^{\prime}-t_{o k}\right)\left(k=1 \ldots N_{M}\right)$ and the same number of electrons in all bunches, $N_{b k}=N_{b}$, then

$$
\sum_{j=1}^{N} e^{i \omega t_{0 j}}=\sum_{k=1}^{N_{M}} \sum_{j=1}^{N_{b k}} e^{i \omega t_{0 j}}=N_{b} M_{b}(\omega) \sum_{k=1}^{N_{M}} e^{i \omega t_{o k}}
$$

Here I replaced the summation over the electrons in each bunch by integration weighted by the bunch pulse-shape function $f\left(t_{0}^{\prime}-t_{o k}\right)$ as in (51), but $f\left(t_{0}^{\prime}-t_{o k}\right)$ is normalized within one period only:

$$
\int_{t_{o k}-\pi / \omega_{b}}^{t_{o k}+\pi / \omega_{b}} f\left(t_{0}^{\prime}-t_{o k}\right) d t_{0}^{\prime}=1 .
$$

The spectral bunching amplitude is then defined by

$$
M_{b}(\omega)=\int_{-\pi / \omega_{b}}^{\pi / \omega_{b}} f\left(t_{0}^{\prime}\right) e^{i \omega t_{0}^{\prime}} d t_{0}^{\prime}
$$

When substituting $t_{o k}=t_{0}-\left(N_{M} / 2-m\right) 2 \pi / \omega_{b}$, where $t_{0}$ is the macropulse center and $m=0,1, \ldots, N_{M}-$ 1 , then (55) can be expressed as

$$
\sum_{j=1}^{N} e^{i \omega t_{0 j}}=N M_{b}(\omega) M_{M}(\omega) e^{i \omega t_{0}},
$$

where $N=N_{b} N_{M}$ is the total number of electrons in the macropulse, and

$$
M_{M}(\omega)=\frac{\sin \left(N_{M} \pi \omega / \omega_{b}\right)}{N_{M} \sin \left(\pi \omega / \omega_{b}\right)}
$$

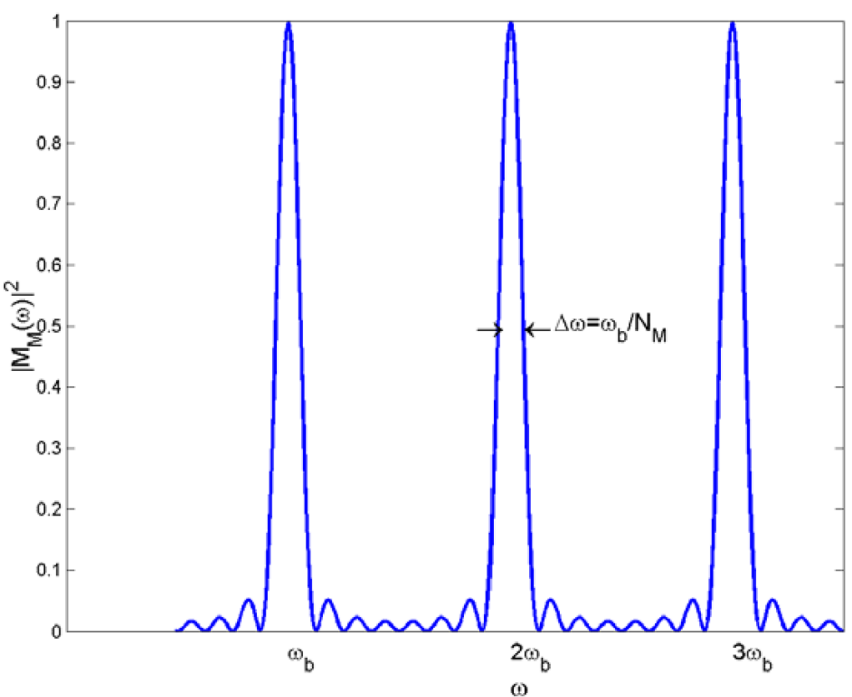

FIG. 9. (Color) The macropulse form-factor function (59) drawn for $N_{M}=8$.

The line-shape function of the macropulse (59), absolute value squared, is shown in Fig. 9. It arrives to its maximal value 1 at frequencies $\omega=n \omega_{b}(n=1,2, \ldots)$. Its bandwidth near frequency $\omega=n \omega_{b}$ is given by

$$
\frac{\Delta \omega}{n \omega_{b}}=\frac{1}{n N_{M}} .
$$

Using (58) in (42) and (43), the expressions for the superradiant and stimulated superradiant spectral energy (48) and (49) get modified into

$$
\begin{aligned}
\left(\frac{d W_{q}}{d \omega}\right)_{\mathrm{SR}}= & \frac{N^{2}}{8 \pi \mathrm{P}_{q}}\left|\Delta \mathrm{W}_{q e}^{(0)}(\omega)\right|^{2}\left|M_{b}(\omega)\right|^{2}\left|M_{M}(\omega)\right|^{2}, \\
\left(\frac{d W_{q}}{d \omega}\right)_{\mathrm{ST}-\mathrm{SR}}= & \frac{N}{2 \pi}\left|C_{q}^{\mathrm{in}}(\omega)\right|\left|\Delta \mathrm{W}_{q e}^{(0)}(\omega)\right|\left|M_{b}(\omega)\right| \\
& \times\left|M_{M}(\omega)\right| \cos \varphi .
\end{aligned}
$$

The macropulse form factor (59) associated with the macropulse duration is usually the dominant narrow bandwidth factor, and its width (60) determines the bandwidth of the spectral energy parameters (61) and (62). The bunchshape function $M_{b}(\omega)$ [Eq. (57)] is always a wide function of $\omega$, and the spectral width of the single electron work function $\Delta \mathrm{W}_{q e}^{(0)}$ is also usually wide (certainly so in CSR). In the case of PB-FEL, the work-function bandwidth narrows down in inverse proportion to $N_{w}$ [Eq. (28)], and thus the macropulse form-factor bandwidth (60) becomes dominant when $n N_{M} \gg N_{w}$.

The fact that the macropusle form factor (59) has multiple peaks of equal amplitude " 1 " at all harmonic frequencies $n \omega_{b}$ is interesting, and may also be of practical significance. It means that it is not essential that the electron beam be bunched at the radiation frequency $\omega$. It can 
be bunched at any subharmonic of the radiation frequency $\omega_{b}=\omega / n$, as long as there is a radiation process at frequency $\omega$ [namely, $\left|\Delta \mathbf{W}_{q e}^{(0)}(\omega)\right|$ does not vanish], and the microbunch has spectral components at this frequency [namely, $\left|M_{b}(\omega)\right|$ does not vanish]. This means, for example, that narrow bandwidth intense $\mathrm{THz}$ radiation can be attained with a train of $e$-beam bunches, if the bunch duration is subpicosecond long but the microbunch repetition frequency may be a subharmonic of the $\mathrm{THz}$ radiation.

If I assume that the macropulse is long enough so that in (61) and (62) the linewidth is determined by (60), then multiplying these equations by $\Delta \omega$ (instead of integration) results in, for the energy emission at harmonic frequency $\omega=n \omega_{b}$,

$$
\begin{aligned}
\left(W_{q}\right)_{\mathrm{SR}} \cong & \frac{N_{M} N_{b}^{2}}{8 \pi \mathrm{P}_{q}} \omega_{b}\left|\Delta \mathrm{W}_{q e}^{(0)}\left(n \omega_{b}\right)\right|^{2}\left|M_{b}\left(n \omega_{b}\right)\right|^{2}, \\
\left(W_{q}\right)_{\mathrm{ST}-\mathrm{SR}} \cong & \frac{N_{b}}{2 \pi} \omega_{b}\left|C_{q}^{\mathrm{in}}\left(n \omega_{b}\right)\right|\left|\Delta \mathrm{W}_{q e}^{(0)}\left(n \omega_{b}\right)\right| \\
& \times\left|M_{b}\left(n \omega_{b}\right)\right| \cos \varphi .
\end{aligned}
$$

The coherent power within the macropulse can be calculated by dividing by the macropulse duration $t_{M}=$ $N_{M} 2 p / \omega_{b}$ :

$$
\begin{aligned}
\left(P_{q}\right)_{\mathrm{SR}} \cong & \frac{1}{16 \pi^{2}} \cdot \frac{N_{b}^{2} \omega_{b}^{2}}{\mathrm{P}_{q}}\left|\Delta \mathrm{W}_{q e}^{(0)}\left(n \omega_{b}\right)\right|^{2}\left|M_{b}\left(n \omega_{b}\right)\right|^{2} \\
\left(P_{q}\right)_{\mathrm{ST}-\mathrm{SR}} \cong & \frac{1}{4 \pi^{2}} \cdot \frac{N_{b} \omega_{b}^{2}}{N_{M}} \cdot\left|C_{q}^{\mathrm{in}}\left(n \omega_{b}\right)\right|\left|\Delta \mathrm{W}_{q e}^{(0)}\left(n \omega_{b}\right)\right| \\
& \times\left|M_{b}\left(n \omega_{b}\right)\right| \cos \varphi .
\end{aligned}
$$

Equations (65) and (66) are also valid in the limit of continuous bunching. Of course, in this case a phasor spectral presentation $f(t)=\operatorname{Re}\left[F(\omega) e^{-i \omega t}\right]$ is more appropriate and would result in similar expressions $[4,38]$.

\section{SUPERRADIANT SPECTRAL ENERGY EMISSION IN PB-FEL AND CSR}

In conclusion of this part I calculate the superradiant spectral energy emission per mode $d W_{q}(\omega) / d \omega$ specifically for PB-FEL and CSR radiation schemes. This is done by substituting the corresponding expressions for the complex work functions $\mathbf{W}_{q e}^{(0)}[(22)$ and (33)] into the generic expression (61). Assuming now a single electron bunch $M_{M}(\omega)=1$, I obtain the following.
For PB-FEL:

$$
\begin{aligned}
\left(\frac{d W_{q}}{d \omega}\right)_{\mathrm{SR}}= & \frac{N_{b}^{2} e^{2} \eta_{q}}{16 \pi} \frac{\left|\mathbf{v}_{\perp 0} \cdot \hat{\mathbf{e}}_{q}^{*}\right|^{2}}{v_{z}^{2}} \\
& \times \frac{L^{2}}{A_{\mathrm{em}}}\left|M_{b}(\omega)\right|^{2} \sin c^{2}(\theta L / 2) \\
= & \frac{N_{b}^{2} e^{2} Z_{q}}{16 \pi}\left(\frac{e B_{w}}{\beta_{z} \gamma m c}\right)^{2} \\
& \times \frac{L^{2}}{k_{w}^{2} A_{\mathrm{em}}}\left|M_{b}(\omega)\right|^{2} \sin c^{2}(\theta L / 2),
\end{aligned}
$$

where $\hat{\mathbf{e}}_{q} \equiv \mathbf{E}_{q}(0,0) /\left|\mathbf{E}_{q}(0,0)\right|$ is a unit vector in the direction of the field of the radiation mode $q, \eta_{q}$ is the mode impedance (in free space and for the fundamental mode of an overmoded waveguide $\eta_{q}=\sqrt{\mu / \varepsilon}$ ), and the effective mode area is defined by

$$
A_{\mathrm{em} q}=\frac{\mathrm{P}_{q}}{\left|\mathbf{E}_{q}(0,0)\right|^{2} /\left(2 Z_{q}\right)} .
$$

In free-space interaction, especially at long wavelengths, the interaction length is limited by diffraction. For estimating the maximum coherent spectral energy, emitted into the fundamental Gaussian mode (16), I assume $L=2 z_{R}$ (18). The effective mode area ("coherence area") is estimated as the waist cross section area at the undulator center:

$$
A_{\mathrm{em}}=\pi w_{0}^{2} / 2=\lambda L / 4 .
$$

This reduces (67) to

$$
\left(\frac{d W_{q}}{d \omega}\right)_{\mathrm{SR}}=\frac{N_{b}^{2} e^{2}}{8 \pi^{2}} \sqrt{\frac{\mu}{\varepsilon}}\left(\frac{e B_{w}}{\beta_{z} \gamma m c}\right)^{2} \frac{L}{k_{w}^{2}} \omega M_{b}(\omega) \sin c^{2}(\theta L / 2) .
$$

For CSR:

$$
\begin{aligned}
\left(\frac{d W_{q}}{d \omega}\right)_{\mathrm{SR}}= & \frac{N_{b}^{2} e^{2} Z_{q}}{4 \pi}\left(\frac{e B_{b}}{\beta \gamma m c}\right)^{2} \frac{L^{4}}{A_{\mathrm{em}}} M_{b}(\omega)\left[\frac{d}{d \theta L}\right. \\
& \times \sin c(\theta L / 2)]^{2}
\end{aligned}
$$

and for a matched free space Gaussian mode (69) [assuming (39) is satisfied]:

$$
\begin{aligned}
\left(\frac{d W_{q}}{d \omega}\right)_{\mathrm{SR}}= & \frac{N_{b}^{2} e^{2}}{2 \pi^{2}} \sqrt{\frac{\mu}{\varepsilon}}\left(\frac{e B_{b}}{\beta \gamma m c}\right)^{2} L^{3} \frac{\omega}{c} M_{b}(\omega) \\
& \times\left[\frac{d}{d \theta L} \sin c(\theta L / 2)\right]^{2} .
\end{aligned}
$$

Despite the formal similarity, the expressions for the PBFEL (67) and (70) and for the CSR (71) and (72) are not straightforwardly comparable. The first has a relatively narrow line-shape function (Fig. 4), centered [for freespace emission (27)] around 


$$
f_{0}=\omega_{0} / 2 \pi=2 \gamma_{z}^{2} c / \lambda_{w},
$$

and the other has a very wide line-shape function (Fig. 5), extending from $f=0$ to $f_{c}=\omega_{c} / 2 \pi \cong 3 \gamma^{2} c / L$, having its maximum value around

$$
f_{m} \cong \omega_{m} / 2 \pi=\gamma^{2} c / L .
$$

Comparing the expressions (70) and (72) for the same wavelength $\lambda_{m}=\left(L / \gamma^{2}\right)_{\mathrm{CSR}}=\left(\lambda_{w} / 2 \gamma_{z}^{2}\right)_{\mathrm{FEL}}$, corresponding to the maximum emission frequencies (73) and (74), the spectral energies (70) and (72) are found similar. This is in good agreement with the intuitive interpretation of CSR as a half-period long FEL, as depicted in Figs. 1(a) and 1(b). However, deriving scaling laws and further comparison between the schemes is not straightforward, and depends on design constraints, and particularly on the frequency range of interest. In principle, for a desirable emission frequency, one would expect enhancement of the radiative emission and narrowing of the spectrum, as the interaction length $L$ is increased by replicating the single bending magnet of CSR [Fig. 1(b)] (namely, producing an undulator structure of longer length, having its half-period equal to the CSR bending magnet length [Fig. 1(a)]). However, these conclusions are modified at long wavelengths by diffraction considerations, and considerations of emission enhancement require then specific design of each radiation scheme.

In PB-FEL and CSR experiments, usually there is pulse repetition-either within the macropulse or continuously. It is instructive to calculate and compare the powers emitted by the repeatedly pulsed $e$-beam in a PB-FEL experiment [39] and a CSR experiment [1]. Besides the different radiation schemes, there is a basic difference between the nature of the bunch repetitions in both cases. In the PBFEL experiment [39], the beam is bunched continuously (within the macropulse) at the frequency of the measured radiation with partial density modulation $\left(M_{b}<1\right)$. Hence, to calculate the power within the macropulse one may use (22) in (65) (with $n=1$ ) and obtain the expression [39]

$$
P_{q}=\frac{M_{b}^{2}}{8} I_{0}^{2} L^{2}\left(\frac{a_{w}}{\gamma \beta_{z}}\right)^{2} \frac{Z_{q}}{A_{\mathrm{em}}} \sin c^{2}(\theta L / 2) .
$$

In [39] maximum power of $25 \mathrm{~W}$ was obtained at $4.5 \mathrm{GHz}$ with a $67 \mathrm{kV}, I_{0}=0.7 \mathrm{~A}$ electron beam with density modulation $M_{b}=0.12$, and a $\lambda_{w}=44.4 \mathrm{~mm}, B_{w}=$ $300 \mathrm{G}, L=0.85 \mathrm{~m}$ wiggler. This measured power agrees well with calculation based on (75).

In the CSR experiment [1], the bunch repetition rate (up to $75 \mathrm{MHz}$ ) is too small, relative to the maximum emission frequency $\sim 1 \mathrm{THz}$, to establish coherence between the bunches. The radiation exhibits, therefore, the entire spectral width of a single bunch. The spectral power is then given by Eq. (72), multiplied by the pulse repetition rate $F_{\text {rep }}$, and the total power would be calculated by integrating this expression over the entire spectral width from $f=0$ to $f_{c}=3 \gamma^{2} c / L$.

Numerical integration of the spectral line-shape function of Fig. 5 gives $\int_{0}^{\infty}\left[\frac{d}{d \bar{\theta}} \sin c(\bar{\theta} / 2)\right]^{2} \bar{\theta} d \bar{\theta} \cong 0$.9. This results in a simple formula for calculating the wideband but spatially coherent CSR power:

$$
P_{q}=\frac{1.8}{\pi^{3}} \frac{\left(N_{b} e\right)^{2}}{\varepsilon_{0}} \gamma^{2} L\left(\frac{e B_{b}}{m c}\right)^{2} F_{\text {rep }}
$$

For the parameters of the CSR experiment of TJL [1], $N_{b} e=100 \mathrm{pC}, \gamma=81, L=0.173 \mathrm{~m}, R=m c / e B_{b}=$ $1.2 \mathrm{~m}, \quad F_{\text {rep }}=37.4 \mathrm{MHz}$, condition (39) is marginally satisfied with $w_{0}$ calculated from condition (18): $X_{j}( \pm L / 2) \cong w_{0}=3 \mathrm{~mm}$. Nevertheless, Eq. (76) predicts $P \sim 6 \mathrm{~W}$, which is close to the power level $P \sim 20 \mathrm{~W}$ reported by the experimentalists.

\section{CONCLUSION}

In this paper I presented a unified formulation and explored the relations between different prebunched electronbeam radiation devices, such as prebunched-beam FEL, coherent synchrotron radiation, and more. All of these prebunched beam radiation schemes can generate enhanced coherent radiation in two different radiative emission processes: superradiance and stimulated superradiance. These processes are more intense than the analogous emission processes in unbunched or partially bunched $e$-beams: spontaneous (shot noise) emission and stimulated emission.

The spectral bandwidth of the radiation from a single electron bunch is usually very wide. It is narrower (and more intense) in the multiple wiggles PB-FEL scheme as compared to the single wiggle scheme of CSR. Narrow spectral bandwidth radiation can be obtained in all $e$-beam radiation schemes, if the electron beam is periodically prebunched. It was shown that the prebunching can be made at subharmonics of the radiation frequency. With developing photocathode $e$-gun technologies, this can help to attain new coherent intense radiation sources in the $\mathrm{THz}$ region.

\section{ACKNOWLEDGMENTS}

I acknowledge helpful discussion with Y. Pinhasi of the College of Judea and Samaria and S. Reiche from UCLA. This work was performed in the Israeli FEL National Knowledge Center supported by the Ministry of Science, Israel, and was supported in part by the Ministry of Infrastructure.

[1] G. L. Carr, M. C. Martin, W. R. McKinney, K. Jordan, and G. R. Neil, Nature (London) 420, 153 (2002).

[2] J. S. Novick and D. S. Saxon, Phys. Rev. 96, 180 (1954). 
[3] F. Curtis Michel, Phys. Rev. Lett. 48, 580 (1982).

[4] I. Schnitzer and A. Gover, Nucl. Instrum. Methods Phys. Res., Sect. A 237, 124 (1985).

[5] F. Ciocci, R. Bartolini, A. Doria, G. P.Gallerano, M. F. Kimmitt, G. Messina, and A. Renieri, Phys. Rev. Lett. 70, 928 (1993).

[6] A. Gover, F. V.Hartemann, G. P. Lesage, N. C. Luhmann, R. S. Zhang, and C. Pelllegrini, Phys. Rev. Lett. 72, 1192 (1994).

[7] B. Faatz, A. A. Fateev, J. Feldhaus, J. Krzywinski, J. Pflueger, J. Rossbach, E. L. Saldin, E. A. Schneidmiller, and M. V. Yurkov, Nucl. Instrum. Methods Phys. Res., Sect. A 475, 363 (2001).

[8] A. Doria, R. Bartolini, J. Feinstein, G. P. Gallerano, and R.H. Pantell, IEEE J. Quantum Electron. 29, 1428 (1993).

[9] M. Cohen, A. L. Eichenbaum, M. Arbel, D. Ben-Haim, H. Kleinman, M. Draznin, A. Kugel, I. M. Yakover, and A. Gover, Phys. Rev. Lett. 74, 3812 (1995).

[10] S. Mayhew, A. I. Al-Shamma'a, R. A. Stuart, A. Shaw, C. Balfour, and J. Lucas, Nucl. Instrum. Methods Phys. Res., Sect. A 393, 356 (1997).

[11] Y. U. Jeong, Y. Kawamura, K. Toyoda, C. H. Nam, and S. S. Lee, Phys. Rev. Lett. 68, 1140 (1992).

[12] M. Asakawa, N. Sakamoto, N. Inoue, T. Yamamoto, K. Mima, S. Nakai, J. Chen, M. Fujita, K. Imasaki, C. Yamanaka, N. Ohigash, T. Agari, T. Asakuma, and Y. Tsunawaki, Nucl. Instrum. Methods Phys. Res., Sect. A 341, 72 (1994).

[13] http://www.frascati.enea.it/thz-bridge/fel-cats.htm

[14] R. Bonifacio, B. W. McNeil, and P. Pierini, Phys. Rev. A 40, 4467 (1989).

[15] A. Gover, A. Friedman, and A. Luccio, Nucl. Instrum. Methods Phys. Res., Sect. A 259, 163 (1987).

[16] N. S. Ginzburg and A. S. Sergeev, Opt. Commun. 91, 140 (1992).

[17] C. P. Neuman, W. S. Graves, and P. G. O'Shea, Phys. Rev. ST Accel. Beams 3, 030701 (2000).

[18] J. H. Brownell, J. Walsh, and G. Ducas, Phys. Rev. E 57, 1075 (1998).

[19] J. R. Neighbors, F. R. Buskirk, and A. Saglan, Phys. Rev. A 29, 3246 (1984).

[20] M. A. Piestrup and P.F. Finman, IEEE J. Quantum Electron. 19, 357 (1983).
[21] W.P. Leemans et al., Phys. Rev. Lett. 91, 074802 (2003).

[22] R. H. Dicke, Phys. Rev. 93, 99 (1954).

[23] S. Krinsky, Phys. Rev. E 59, 1171 (1999).

[24] S. M. Wiggins, D. A. Jaroszynski, B. W. J. McNeil, G. R. M. Robb, P. Aitken, A. D. R. Phelps, A. W. Cross, K. Ronald, N. S. Ginzburg, V. G. Shpak, M. I. Yalandin, S. A. Shunailov, and M. R. Ulmaskulov, Phys. Rev. Lett. 84, 2393 (2000).

[25] B. W. J. McNeil, G. R. M. Robb, and D. A. Jaroszynski, Opt. Commun. 165, 65 (1999).

[26] Y. Pinhasi and Y. Lurie, Phys. Rev. E 65, 026501 (2002).

[27] D. A. G. Deacon, L. R. Elias, J. Madey, G. J. Ramian, H. A. Schwettman, and T. I. Smith, Phys. Rev. Lett. 38, 892 (1977).

[28] A. Gover , H. Freund, V.L. Granatstein, J. H. McAdoo, and C. M. Tang, Infrared and Millimeter Waves, edited by K. Button (Academic, New York, 1984), Vol. 11, p. 291.

[29] H. Motz, W. Thon, and R. N. Whitehurst, J. Appl. Phys. 24, 826 (1953).

[30] Y. Shibata, T. Takahashi, T. Kanai, K. Ishi, M. Ikezawa, J. Ohkuma, S. Okuda, and T. Okada, Phys. Rev. E 50, 1479 (1994).

[31] J. G. Neumann, P. G. O'Shea, D. Demske, W. S. Graves, B. Sheehy, H. Loos, and G. L. Carr, Nucl. Instrum. Methods Phys. Res., Sect. A 507, 498 (2003).

[32] See, e.g., H. Kogelnik, in Guided Wave Optoelectronics, edited by T. Tamir (Springer-Verlag, Berlin, 1988), Chap. 2.

[33] A. Yariv, Quantum Electronics (Wiley, New York, 1988).

[34] S. Krinsky, IEEE Trans. Nucl. Sci. 30, 3078 (1983); G. K. Green, Brookhaven National Laboratory Report No. 50522, 1976.

[35] J. Schwinger, Phys. Rev. 75, 1912 (1949).

[36] G. Geloni, E. L. Saldin, E. A. Schneidmiller, and M. V. Yurkov, DESY Report No. 03-031, 2003.

[37] S. Reiche and A. Gover (unpublished).

[38] M. Arbel, A. Abramovich, A. L. Eichnebaum, A. Gover, H. Kleinman, Y. Pinhasi, and I. M. Yakover, Phys. Rev. Lett. 86, 2561 (2001).

[39] M. Arbel, A. L. Eichenbaum, Y. Pinhasi, Y. Lurie, M. Tecimer, A. Abramovich, H. Kleinman, I. M. Yakover, and A. Gover, Nucl. Instrum. Methods Phys. Res., Sect. A 445, 247 (2000). 